\title{
Possibility of amoebas' aggregation in finite time
}

\author{
Saïd HILMI and Chérif ZITI
}

\begin{abstract}
The dynamic of amoebas in favorable circumstances is modeled by a nonlinear system of Partial Differential Equations arising in chemotaxis. The competition between different parameters of this system plays a major role in the process of aggregation. Throughout this paper, we prove the existence of self-similar solutions that blow up in finite time in a dimensional space and under specific circumstances depending upon the position of those parameters.
\end{abstract}

\section{Introduction}

Many fundamental processes in biology and physiology depend on the ability of cells reaction to external cues. A reaction to chemical substances is called Chemotaxis. This can lead to a biased motion of some organisms (such as amoebae, bacteria, endothelial cells, etc...) towards the gradients of chemicals concentration. The purpose is to provide a more favorable environment for their survival. Such a movement along the gradient of the chemical substance can be either positive chemotaxis where organisms move toward a higher concentration or negative chemotaxis where they move away from it.

From a Partial Differential Equation viewpoint, the description of biological complex systems is usually carried out based upon the following perspectives:

Key Words: Dynamic Systems, Partial Differential Equations, Blow-up, Chemotaxis, Self-similar solutions.

2010 Mathematics Subject Classification: Primary 35B44, 35C06; Secondary 92C17.

Received: September, 2011.

Revised: January, 2012.

Accepted: April, 2012. 
The microscopic perspective considers a more local (say individual) or mesoscopic level which involves kinetic (Boltzmann type) equations with nonlinear scattering kernels. The arguments used to describe the microscopic motion are supported by different approaches such as Fourier's law, Fick's law, biased random walk, interacting particle systems, transport equations, stochastic processes and multi-phase flow modeling; see [15] and the references therein.

The macroscopic perspective considers the whole population density involved in the process. It is no more concerned with the individual microscopic data but rather with global properties where relations settle between various macroscopic quantities. They are usually formulated and derived from microscopic laws; see [10] for more details.

In accordance with the latter approach, different mathematical models have been suggested to describe the chemotaxis process. Nevertheless, the one provided by Keller and Segel (K-S) remains the most prominent and widely studied; see $[11,12,13,16]$ for various assessments. The K-S model is basically used to describe the aggregation of the slime mold amoebae Dictyostelium discoideum $[3,9]$. It has also been successfully used in various contexts such as angiogenesis to describe the phenomenon of sprouting of new blood vessels and the growth of a tumor [4]. This was accomplished thanks to the model capacity to replicate key behavior of chemotactic populations and its ability to demonstrate aggregation in certain parameter regions.

In this paper, we shall consider the following parabolic-parabolic K-S system for any $t<0, x \in \mathbb{R}^{n}$

$$
\left\{\begin{array}{l}
\frac{\partial U}{\partial t}+\nabla(U \chi(S) \nabla S)=\mu \Delta U \\
\frac{\partial S}{\partial t}=K(S) U+\nu \Delta S \\
\chi(S)=\delta S^{-\alpha}, K(S)=k S^{m} \\
\mu, \delta, k>0 \text { and } \alpha, m, \nu \geq 0
\end{array}\right.
$$

where it is assumed that the evolution of the concentration of amoebae $U(x, t)$ and the concentration $S(x, t)$ of substrate are governed respectively by two equations:

The first equation expresses the amoebas conservation where the term on the right represents the motion in the absence of chemotaxis. Thus, with $\nabla S=0$ the equation becomes identical to the diffusion equation where $\mu$ represents 
the motility coefficient. The second term on the left represents the flux due to chemotaxis where $\chi(S)$ represents a measure of the chemotaxis strength and is, hence, termed the chemotactic sensitivity while $\delta$ represents the chemotactic coefficient.

The second equation describes the substrate conservation that is assumed to diffuse with a coefficient of diffusion $\nu$ and is produced by the amoebae with the rate $K(S)$.

Under certain formulations of the above model, the phenomenon of aggregation has been shown to lead to finite-time blow-up, and a large body of work has been devoted to determine when blow-up occurs or whether globally solutions exist. Indeed, under various assumptions, it has been rigorously proven that for one dimension, the solutions exist globally. For higher dimensions, there exists a threshold phenomenon with blow-up solutions. In two space dimension, which is a particular case, the finite time blow-up solution exist when the threshold is above a certain critical mass; see e.g. [6] and $[10,15]$. The threshold advanced here is located between the diffusion dominated regime and the regime of aggregation.

On the other hand, much interest has been aroused by the forms of solutions of the system (1) and their behavior change in different aspects. In this article, the emphasis is directed to self-similar solutions. Such solutions can be obtained by integrating (1) on the interval $\left[t_{0}, t\right]$ for any $0<t_{0}<t$, that is, solutions of

$$
\begin{gathered}
U(t, .)=\exp ^{\left(t-t_{0}\right) \Delta} U\left(t_{0}, .\right)-\int_{t_{0}}^{t}\left(\nabla \exp ^{(t-z) \Delta}\right) \cdot(U(z, .) \nabla S(z, .)) d z, \\
S(t, .)=\exp ^{\frac{t-t_{0}}{\tau} \Delta} S\left(t_{0}, .\right)+\frac{1}{\tau} \int_{t_{0}}^{t} \exp ^{\frac{t-z}{\tau} \Delta} U(z, .) d z
\end{gathered}
$$

for the particular case $\mu=\chi(s)=1$ and $K(s)=\nu=\frac{1}{\tau}$. We refer to $[6,14]$ for this and additional results.

Alternatively, we can prove the existence of self-similar solutions in the same spirit as in Childress and Percus [5], i.e, solutions which can be written as

$$
U(x, t)=(-t)^{a} u(\zeta), \quad S(x, t)=(-t)^{b} s(\zeta)
$$

where

$$
x=\left(x_{1}, x_{2}, \ldots, x_{n}\right), \quad \zeta=\frac{|x|^{2}}{t}=\frac{\sum_{i=1}^{n} x_{i}^{2}}{t},
$$


Such self-similar solutions are sufficiently smooth (at least integrable) and preserve mass since $a=-\frac{n}{2}$. It remains to determine in which case their evolution lead to the blow up into a set of $\delta$-functions. These $\delta$-functions arise as a result of the competition between $m$ and $\alpha$, which express respectively the depletion rate of the substrate and the strength of the chemotactic attraction.

For instance in the case of the bacteria system $(k<0)$, it has been shown in [18] that the bacterial density is concentrated in finite time at the origin in one space dimension for $\nu=0$. For two space dimensions and initial data for the bacterial density which is zero at the origin, chemotactic rings are concentrated around the origin after finite time. In higher space dimensions, blow-up of the solution occurs by an initial singularity of the chemoattractant in the origin. For $\nu>0$, there exist smooth initial data leading to finite time blow-up of the solution in one space dimension.

Using the same technical tools introduced in [18] and considering the system (1) with a smooth initial data $t=t_{0}<0$, we investigate whether solutions (2) blow up at $x=0$ and $t=0$ for the non-diffusive case $\mu=0$, since $a=-\frac{n}{2}$ and $b=\frac{n-2}{2(m-1)}$. Our findings are stated as follows:

Assuming that the diffusion of substrate has a small effect compared to that of amoebae (i.e. $\nu$ is taken to be zero) and $m$ ranges in the interval $] 0,1[$, we can prove that for $\alpha=1$ the aggregation of amoebae will be possible in a dimensional space higher than two. For $\alpha>1$, the same result holds true in dimension two. In the case of $\alpha=m$, we construct radially symmetric solutions that blow up as a chemotactic collapse in finite time.

For $\nu$ considered very large (therefore the term $\frac{\partial S}{\partial t}$ of the second equation of (1) can be neglected), the substrate satisfies a quasi-static elliptic PDE. In this case, we reformulate radially symmetric solutions under a suitable scaling that follow Burger's equation. This can lead to a blow-up as $\delta$-function (amoebae aggregation) at the proximity of the origin in dimension one, and on a ball in a multidimensional space.

The paper is structured as follows. In Section 2 we state the scaling (2) in detail and some preliminary results that allows in the sequel to investigate the possibility of aggregation according to the position of $\alpha$ and $m$. In Section 3 and resp. Section 4, we will study the non diffusive system (1) for the case $\alpha>m$ where $\alpha>1$ (resp. $\alpha<1$ ). Section 5 is devoted to the case of $\alpha=m$. In Section 6, we will study the case when diffusion of chemicals takes place 
much faster than that of the species.

\section{Preliminaries}

The scaling (2) can be justified in the following way :

First, we consider $t_{0}<t<0$ instead of $0<t_{0}<t$ since (1) is autonomous. From a smooth initial data $t=t_{0}<0$, we study the possibility of blow-up at the point $x=0$ and the time $t=0$ in the full space $\mathbb{R}^{n}$ without boundary conditions. With additional assumptions on initial data, the conservation of mass for the density function and the nonnegativity of density function and chemo-attractant concentration are assured, i.e.,

$$
\int_{\mathbb{R}^{n}} u(., t)=\int_{\mathbb{R}^{n}} u_{0}(., t)=M, \quad \forall t \leq 0, \quad u(., t) \geq 0, \quad s(., t) \geq 0 .
$$

Then, we consider the case $\nu=0$, whenever the diffusion of substrate is small compared to the motility of the species of density $\mu$, in $n$ space dimensions of (1), namely,

$$
\begin{gathered}
U_{t}+\delta \sum_{i=1}^{n}\left(U S^{-\alpha} S_{x_{i}}\right)_{x_{i}}=\mu \Delta U \quad x \in \mathbb{R}^{n}, t_{0}<t<0, \\
S_{t}=k S^{m} U .
\end{gathered}
$$

The magnitude of $U(x, t)$ in aggregate in $\mathbb{R}^{n}$ will be $O\left(\delta^{-n}\right)$, where $\delta$ is an aggregate dimension which decreases to zero when a collapse occurs. Thus, if we take $S=\delta^{-\gamma}$ and $-t \sim \delta^{\epsilon}$, then $\frac{\partial}{\partial t} \sim \delta^{-\epsilon}$ and $\nabla \sim \delta^{-1}$. With respect to dependance on $\delta$, the equations on (3) and (4) then take the following form:

$$
\left\{\begin{array}{l}
\left(\delta^{-n-\epsilon}, \delta^{-n-2}, \delta^{-n-2}\right)=0 \\
\left(\delta^{-\epsilon+\gamma}, \delta^{-n+\gamma m}\right)=0
\end{array}\right.
$$

Therefore, if we choose

$$
\epsilon=2, \quad \gamma=\frac{2-n}{1-m}=2 b
$$

all terms are then comparable in each equation, and there is a possibility of blowing up for each scaling in any space dimension. According to (5) and (6), 
a spatial scale of $\delta$ implies a temporal scale of $\delta^{2}$.

In addition, assuming that the blow up occurs at finite time $t=0$ we introduce the scaled variable

$$
\zeta=\frac{|x|^{2}}{t}=\frac{\sum_{i=1}^{n} x_{i}^{2}}{t} \sim 1, \quad t<0 .
$$

Hence, since $S=\delta^{-\gamma} \sim(-t)^{b} \delta$, the scaling of $S$ in (2) is followed. Note that,

$$
\forall t \in\left(t_{0}, 0\right)\left\{\begin{array}{l}
\int_{\mathbb{R}^{n}} U(x, t) d x=M \\
\lim _{|x| \rightarrow+\infty} S(x, t)=\lim _{\zeta \rightarrow-\infty} s(\zeta)=0
\end{array}\right.
$$

Let $(U(x, t), S(x, t))$ be the solution to (3), (4) and (7). The equation (7) implies

$$
a=-\frac{n}{2}, \quad \int_{-\infty}^{0} u(\zeta) \rho(\zeta) d \zeta=2 \omega_{n}^{-1} M
$$

where $\omega_{n}$ is the unit ball volume and $\rho(\zeta)=(-\zeta)^{\frac{n}{2}-1}$.

Lemma 2.1. Let $(u(\zeta), s(\zeta))$ be defined in (2). Assume that

$$
a=-\frac{n}{2}, \quad b(1-\alpha)=0, \quad b=\frac{n-2}{2(m-1)}
$$

then

$$
\left\{\begin{array}{l}
\frac{d}{d \zeta}\left[(-\zeta)^{-a} u\right]=\frac{d}{d \zeta}\left[4 \delta(-\zeta)^{\frac{n}{2}} u s^{-\alpha} \dot{s}-4 \mu(-\zeta)^{\frac{n}{2}} \dot{u}\right] \\
b s-\zeta \dot{s}=-k s^{m} u
\end{array}\right.
$$

where $(\cdot)$ stands for $\frac{d}{d \zeta}$

Lemma 10 can be proved in a similar way as in [18], so we omit the proof here.

Remark 2.2. From (9), we have

$$
b(1-\alpha)=0 \Leftrightarrow b=0 \quad \text { or } \quad \alpha=1
$$

So, assuming that $m<1$ then

- $b>0$ if and only if $n<2$.

- If $\alpha=1$ then any negative parameter $b$ is possible.

- If $\alpha \neq 1$ then $b=0$, hence $n=2$. 
3 Blow-up for $m<\alpha=1$ and $\mu=0$

Assuming that $\alpha=1$ since $a=-\frac{n}{2}$. By integrating the first equation of (10) in $\mathbb{R}_{-}$, we obtain

$$
(-\zeta)^{\frac{n}{2}} u=4 \delta(-\zeta)^{\frac{n}{2}} u s^{-1} \dot{s}-4 \mu(-\zeta)^{\frac{n}{2}} \dot{u}+C
$$

for some constant $C$.

Notice that taking $\zeta=0$, we can observe that $C=0$. Thus, from Lemma 2.1, we deduce the following proposition

Proposition 3.1. Assume that $\left.\left.u \in C^{2}(]-\infty, 0\right]\right)$ and $\left.\left.s \in C(]-\infty, 0\right]\right)$, as defined in (2). Then the system in (3) and (4) is equivalent to

$$
\left\{\begin{array}{l}
u=4 \delta u s^{-1} \dot{s}-4 \mu \dot{u} \\
b s-\zeta \dot{s}=-k s^{m} u
\end{array}\right.
$$

The problem is thus reduced to the form (12). This allows us to prove

Lemma 3.2. Assume that $a, b, m, \alpha$ satisfy (9) with $m<\alpha=1$ and $\mu=0$. Then for any positive $A$ and $\zeta_{0}=4 \delta b$, the general solution of (12) is

$$
\begin{gathered}
u(\zeta)=\left\{\begin{array}{lr}
-\frac{A^{1-m}}{k}\left(b-\frac{\zeta}{4 \delta}\right) \exp \left(\frac{1-m}{4 \delta} \zeta\right) & \text { if } \zeta>\zeta_{0} \\
0 & \text { otherwise }
\end{array}\right. \\
s(\zeta)=\left\{\begin{array}{lr}
A \exp \left(\frac{\zeta}{4 \delta}\right) & \text { if } \zeta>\zeta_{0} \\
A\left(\frac{\zeta}{\zeta_{0}}\right)^{b} \exp \left(\frac{\zeta_{0}}{4 \delta}\right) & \text { otherwise }
\end{array}\right.
\end{gathered}
$$

Proof. For $\mu=0$, we rewrite (12)

$$
\left\{\begin{array}{l}
u=4 \delta s^{-1} \frac{d s}{d \zeta} u \\
b s-\zeta \frac{d s}{d \zeta}=-k s^{m} u
\end{array}\right.
$$

- If $u \neq 0$, then for $\zeta>\zeta_{0},(13)$ and (14) can be derived by dividing the first equation of (15) by $u$ and the second by $s$.

- If $u \equiv 0$ at least on some interval $I$, then on this interval $s(\zeta)=$ $B(-\zeta)^{b}$, for some positive constant $B$. We can easily check that $B=$ $A\left(-\zeta_{0}\right)^{-b} \exp \left(\frac{\zeta_{0}}{4 \delta}\right)$. 

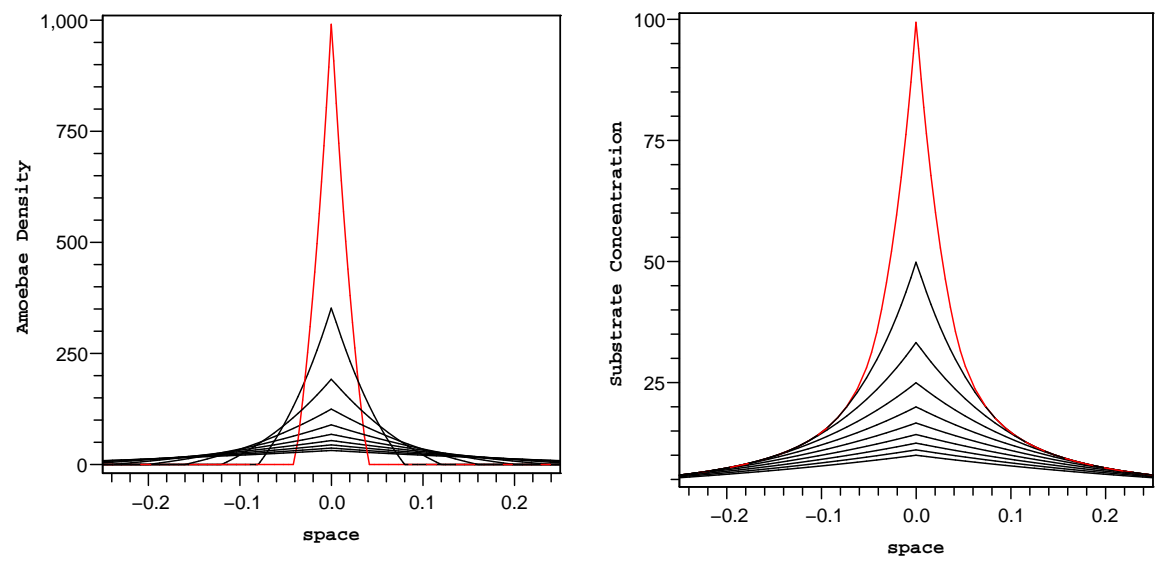

Figure 1: The concentration in amoebae (left) (resp. in chemoattractant (right)) obtained for $\alpha=1, m=0.5, n=3, k=1, \delta=1$ and for ten successive values of time $t_{0}=-0.1<t_{1}<\ldots<t_{9}=-0.01$ with time-step $\Delta t=0.1$. the initial data, for which $t=t_{0}=-0.1$, corresponds in each figure to the curve with the lowest peak.

Remark 3.3.

- The function u defined in (13) is integrable on ] - $\infty, 0]$;

- $\lim _{\zeta \rightarrow-\infty} u(\zeta)=0$ if and only if $m<1$.

So, starting with initial data

$$
U\left(x, t_{0}\right)=U_{0}(x)=\left(-t_{0}\right)^{a} u\left(\frac{|x|^{2}}{t_{0}}\right), \quad S\left(x, t_{0}\right)=S_{0}(x)=\left(-t_{0}\right)^{b} s\left(\frac{|x|^{2}}{t_{0}}\right),
$$

there exists $(U, S)$ solution of (1) that blows up at the origin at the time $t=0$. The shapes in Figure 1 illustrate a rapid evolution to amoebae density solution into blow-up as a delta-function. Note that $a=-\frac{n}{2}$. Therefore, the blow up of $U$ is possible since the total mass $M$ is constant.

Corollary 3.4. Under the assumptions of Lemma 3.2, let $n>2$ (i.e $b<0$ ) 
there then exists a non negative regular solution

$$
(u(\zeta), s(\zeta))= \begin{cases}\left(-\frac{A^{1-m}}{k}\left(b-\frac{\zeta}{4 \delta}\right) \exp \left(\frac{1-m}{4 \delta} \zeta\right), A \exp \left(\frac{\zeta}{4 \delta}\right)\right) & \text { if } \zeta>\zeta_{0} \\ \left(0, B(-\zeta)^{b}\right) & \text { otherwise }\end{cases}
$$

where $\zeta_{0}=4 \delta b$.

On $\left.]-\infty, \zeta_{0}\right], A$ and $B$ are chosen such as $\left.s(\zeta)\right)$ is continuous in $\zeta=\zeta_{0}$.

Remark 3.5. $s$ is not derivable in $\zeta=\zeta_{0}$, but the first equation of (3) is correct because $u\left(\zeta_{0}\right)=0$, hence $\frac{u}{s} \nabla s=0$ in $\zeta=\zeta_{0}$. In [13, 18] the diffusion coefficient of substrate $\nu$ is taken to be zero because the diffusion of the substrate has a small effect compared to the diffusion of the amoebae. It means that $(u(\zeta), s(\zeta))$ is approximated uniformly by $\left(u_{\nu}, s_{\nu}\right)$ when $\nu$ is sufficiently small. This will be proved in the following lemma

Lemma 3.6. Assume that $a=-\frac{n}{2}, \alpha=1, \mu=0$ and $\nu>0$. Then the solution of the system (1) with a diffusion of substrate $\nu(\nu<<1)$ is $\left((-t)^{a} u_{\nu}(\zeta),(-t)^{b} s_{\nu}(\zeta)\right)$ where

$u_{\nu}(\zeta)= \begin{cases}-\frac{A^{1-m}}{k}\left[b-\frac{\zeta}{4 \delta}-\nu\left(\frac{n}{2 \delta}+\frac{\zeta}{4 \delta^{2}}\right)\right] \exp \left(\frac{1-m}{4 \delta} \zeta\right) & \text { if } \zeta>\left(b-\frac{n \nu}{2 \delta}\right)\left(\frac{1}{4 \delta}+\frac{\nu}{4 \delta^{2}}\right) \\ 0 & \text { otherwise }\end{cases}$

$$
s_{\nu}(\zeta)= \begin{cases}A \exp \left(\frac{\zeta}{4 \delta}\right) & \text { if } u(\zeta) \neq 0 \\ B(-\zeta)^{b} & \text { otherwise }\end{cases}
$$

and

$$
u_{\nu}-u=O(\nu), \quad s_{\nu}=s
$$

Proof. The system with a diffusion of substrate is rewritten as

$$
U_{t}+\delta \sum_{i=1}^{n}\left(U S^{-\alpha} S_{x_{i}}\right)_{x_{i}}=\mu \Delta U S_{t}=k S^{m} U+\nu \Delta S
$$

Using the same computation as in the previous section, we have

$$
\left\{\begin{array}{l}
\frac{d}{d \zeta}\left[(-\zeta)^{-a} u_{\nu}\right]=(-\zeta)^{-a-\frac{n}{2}} \frac{d}{d \zeta}\left[4 \delta(-\zeta)^{\frac{n}{2}} u_{\nu} s_{\nu}^{-\alpha} \dot{s_{\nu}}\right] \\
b s_{\nu}-\zeta \dot{s_{\nu}}=-k s_{\nu}^{m} u_{\nu}+\nu\left(2 n \dot{s_{\nu}}+4 \zeta \ddot{s_{\nu}}\right)
\end{array}\right.
$$

which yields after integration

$$
u_{\nu}=-A^{1-m} k^{-1}\left[b-\frac{\zeta}{4 \delta}-\nu\left(\frac{n}{2 \delta}+\frac{\zeta}{4 \delta^{2}}\right)\right] \exp \left(\frac{1-m}{4 \delta} \zeta\right)
$$


So, if $u_{\nu} \neq 0$ then

$$
u_{\nu}-u=\nu A^{1-m}(2 k \delta)^{-1}\left(n+\frac{\zeta}{2 \delta}\right) \exp \left(\frac{1-m}{4 \delta} \zeta\right) s_{\nu}=s
$$

and

$$
\sup _{\zeta<0}\left|u_{\nu}(\zeta)-u(\zeta)\right|=\nu A^{1-m}(k(m-1) \delta)^{-1} \exp \left(\frac{n(m-1)}{2}-1\right)
$$

Conclusion 3.7. If $\alpha=1, \mu=0$ then aggregation will be possible if $b<0 \Leftrightarrow$ $n>2$ because the solution $u(\zeta)$ of (13) will not be negative.

4 Blow-up for $m<1, \alpha \neq 1$ and $\nu=\mu=0$

From Remark 2.2, we have seen that $b=0$, hence $n=2$. In this case, we set the following lemma

Lemma 4.1. Under the assumptions $n=2, a=-1$ and $\mu=0$, the general solution of $(10)$ is

$$
\left\{\begin{array}{l}
u(\zeta)=\frac{\zeta}{4 k \delta}\left[\frac{1-\alpha}{4 \delta} \zeta+s_{0}^{1-\alpha}\right]^{\frac{\alpha-m}{1-\alpha}} \\
s(\zeta)=\left[\frac{1-\alpha}{4 \delta} \zeta+s_{0}^{1-\alpha}\right]^{\frac{1}{1-\alpha}}
\end{array}\right.
$$

where $s_{0}$ is constant.

It's obvious to prove that

$$
\begin{array}{ll}
\lim _{\zeta \rightarrow-\infty} u(\zeta)=0 & \text { if and only if } m<1 \\
\lim _{\zeta \rightarrow-\infty} s(\zeta)=0 & \text { if and only if } \alpha>1
\end{array}
$$

A typical situation is illustrated in Figure 2 and on 3D in Figure 3; the peaks of the displayed concentration of substrate $S(x, t)$ correspond to delta function type singularities of the particle density $U(x, t)$ 

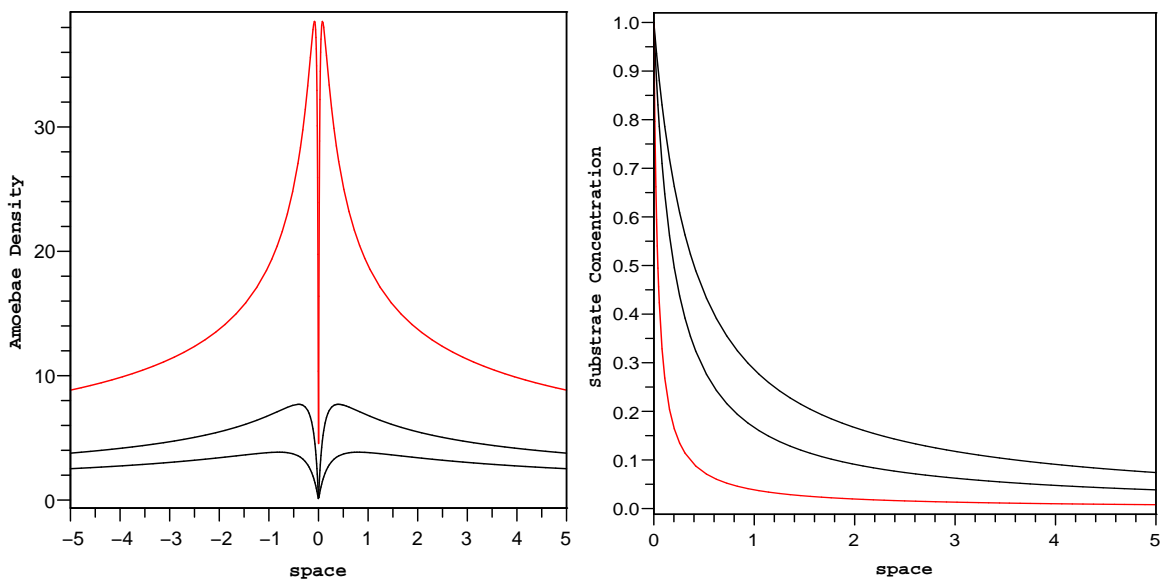

Figure 2: The density of amoebae (left) and the concentration of substrate (right) obtained for $\alpha=2, m=0.5, n=2, \delta=1$ and $t_{0}=-0.1<t_{1}=$ $-0.05<t_{2}=-0.01$ (in red). The bi-dimensional of amoebae profiles illustrate 2 peaks rather than in multi-dimensional space.

Proof. We rewrite (10)

$$
\left\{\begin{array}{l}
\frac{d}{d \zeta}\left[(-\zeta)^{-a} u\right]=\frac{d}{d \zeta}\left[4 \delta \zeta u s^{-\alpha} \dot{s}\right] \\
-\zeta \dot{s}=-k s^{m} u
\end{array}\right.
$$

Integrating the first equation where $a=-1$ we obtain,

$$
-\zeta u=4 \delta \zeta u s^{-\alpha} \dot{s}+C
$$

Where choosing $\zeta=0$, we can see that $C=0$.

Then dividing by $-\zeta u$, we find

$$
1=4 \delta s^{-\alpha} \dot{s}
$$

This becomes where integrating for $\alpha \neq 1$

$$
\frac{s^{1-\alpha}}{1-\alpha}-\frac{s_{0}^{1-\alpha}}{1-\alpha}=\frac{\zeta}{4 \delta}
$$

Combining (19) with the second equation of $(18)$, we obtain $s(\zeta)$. 

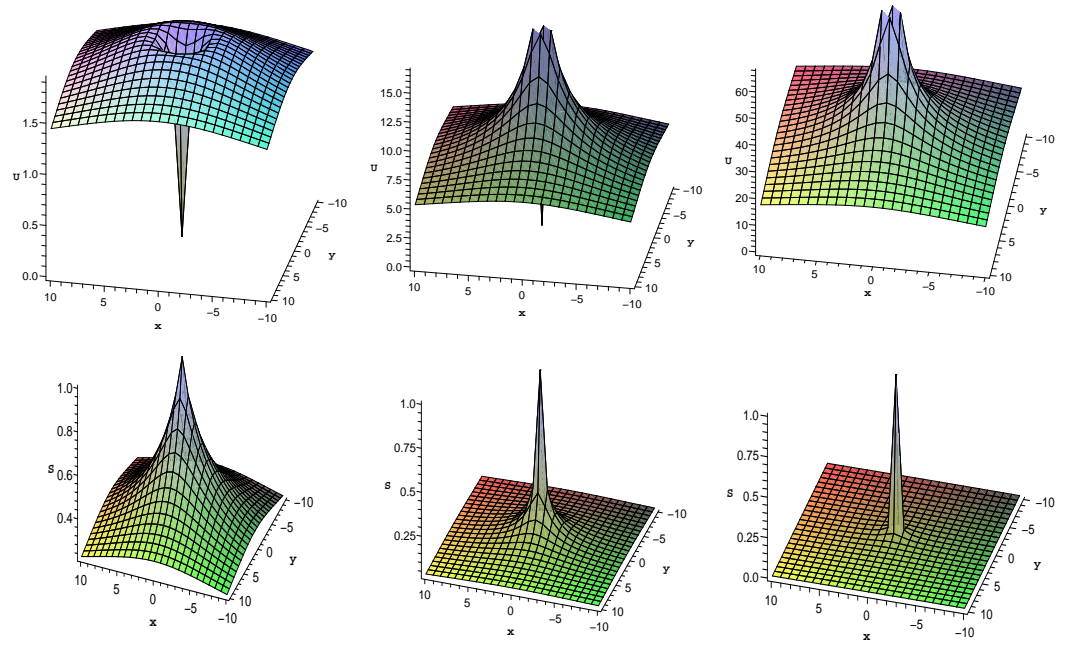

Figure 3: The amoebae density " $U$ " and the concentration of substrate "S" plotted on $3 D$ for $\alpha=2, m=0.5, n=2, \delta=1$ and $t_{0}=-0.1<t_{1}=-0.01<$ $t_{2}=-0.001$.

Conclusion 4.2. In the bi-dimensional non-diffusive case $n=2 \Leftrightarrow b=0$, the aggregation will be possible if $m<1$ and $\alpha>1$.

\section{Blow-up for $m=\alpha<1$ and $\nu=0$}

In this section, we shall investigate radial solutions of (1) that come to a blow up result in finite time as a chemotactic collapse. One shall show that this phenomenon is only possible if the dimensional space $n>2$. For that, with the transformation

$$
S= \begin{cases}\frac{s^{1-m}}{1-m} & \text { if } m<1 \\ \ln s & \text { if } m=1\end{cases}
$$

we rewrite the system (1) as

$$
\left\{\begin{array}{l}
U_{t}+\delta \nabla(U \nabla S)=\mu \Delta U \\
S_{t}=k U
\end{array}\right.
$$


which is a particular case $\left(k_{0}=\nu=0\right)$ of the system studied in Childress and Percus [5]

$$
\left\{\begin{array}{l}
U_{t}+\delta \nabla(U \nabla S)=\mu \Delta U \\
S_{t}=k U-k_{0} S+\nu \Delta S
\end{array}\right.
$$

Note that to prevent $S$ from been negative, the scaling of Childress and Percus cannot be used unless $m<1$.

Proposition 5.1. If $n>2$ then there exists self similar solutions of (20) that one possibly have aggregation in finite time. These are given by introducing the transformation behavior

$$
\left\{\begin{array}{l}
R=(-t)^{\frac{-2}{n+2}} r \\
U=U(r, t)=(-t)^{\frac{-2 n}{n+2}} U_{1}(R) \\
S=S(r, t)=(-t)^{-\frac{n-2}{n+2}} S_{1}(R)
\end{array}\right.
$$

where

$$
U_{1}(R)= \begin{cases}\frac{1}{\delta k(n+2)}\left(R_{0}^{2}-R^{2}\right) & \text { if } R \leq R_{0} \\ 0 & \text { otherwise }\end{cases}
$$

and

$$
S_{1}(R)= \begin{cases}\frac{1}{\delta(n+2)}\left(\frac{n+2}{n-2} R_{0}^{2}-R^{2}\right) & \text { if } R \leq R_{0} \\ \frac{4}{\delta\left(n^{2}-4\right)}\left(\frac{R_{0}}{R}\right)^{\frac{n-2}{2}} & \text { otherwise }\end{cases}
$$

Proof. We introduce the transformations given by the formulas (21). $U_{1}$ and $S_{1}$, been bounded and regular functions, we compute

$$
\frac{\partial R}{\partial t}=\frac{2}{(n+2)} \frac{1}{(-t)} R, \quad \frac{\partial R_{i}}{\partial r_{i}}=(-t)^{-\frac{2}{n+2}}
$$

Substituting (20) in (21), the system for $U_{1}$ and $S_{1}$ becomes

$$
\left\{\begin{array}{l}
\left(\frac{2 n}{n+2}+\frac{2}{n+2} R \cdot \nabla_{R}\right) U_{1}(R)+\delta \nabla_{R} \cdot\left(U_{1} \nabla_{R} S_{1}\right)=(-t)^{\frac{n-2}{n+2}} \mu \Delta_{R} U_{1}(R) \\
\left(\frac{n-2}{n+2}+\frac{2}{n+2} R \cdot \nabla_{R}\right) S_{1}(R)-k U_{1}(R)=0
\end{array}\right.
$$

which turn out to be if $t \rightarrow 0^{-}$since $n>2$

$$
\left\{\begin{array}{l}
\frac{2}{\delta(n+2)} \nabla_{R}\left(R U_{1}\right)+\nabla_{R} \cdot\left(U_{1} \nabla_{R} S_{1}\right)=0 \\
\left(n-2+2 R \cdot \nabla_{R}\right) S_{1}=k(n+2) U_{1}
\end{array}\right.
$$


The first equation in (23) has the particular integral solution

$$
U_{1}\left[\Delta_{R} S_{1}+\frac{2 R}{\delta(n+2)}\right]=0
$$

So,

$$
\begin{cases} & S_{1}=K-\frac{R^{2}}{\delta(n+2)} \\ \text { or } & \\ & U_{1}=0\end{cases}
$$

By substituting (25) in the second equation of (23), one obtain

$$
\begin{cases}\text { or } & U_{1}=\frac{1}{\delta k(n+2)}\left[(n-2) \delta K-R^{2}\right] \\ & S_{1}=K^{\prime} R^{-\frac{n-2}{2}}\end{cases}
$$

Remark 5.2. It's clear that $U_{1}$ tends to be null before $S_{1}$. Now we let $K=$ $\frac{R_{0}^{2}}{\delta(n-2)}$, and valued $K^{\prime}$ by continuity. Finally, we have

$$
\begin{gathered}
\left\{\begin{array}{cl}
U_{1}=\frac{1}{\delta k(n+2)}\left(R_{0}^{2}-R^{2}\right) & \text { if } R \leq R_{0} \\
U_{1}=0 & \text { otherwise }
\end{array}\right. \\
\begin{cases}S_{1}=\frac{1}{\delta(n+2)}\left(\frac{n+2}{(n-2)} R_{0}^{2}-R^{2}\right) & \text { if } R \leq R_{0} \\
S_{1}=\frac{4}{\delta\left(n^{2}-4\right)}\left(\frac{R_{0}}{R}\right)^{\frac{n-2}{2}} & \text { otherwise }\end{cases}
\end{gathered}
$$

Remark 5.3. Since the totality of population is conserved we have

$$
M=\int U(r, t) d r^{n}=\int U_{1}(R) d R^{n}=\frac{2 S_{n}}{(n+2)^{2} \delta k} R_{0}^{2}
$$

$S_{n}$ been the $n$ dimensional unit ball volume, this allows to compute $R_{0}$

$$
R_{0}=(n+2) \sqrt{\frac{k M \delta}{2 S_{n}}}
$$




\section{6 blow-up for $\mu=0$ and $\nu>>1$}

Assuming $\nu$ very large means that the diffusion of the chemical takes place much faster than that of the species. This leads to neglect the term of $\frac{\partial S}{\partial t}$. As a consequence, the second equation of (1) becomes $-\nu \Delta S=U$.

Therefore we now consider the following parabolic-elliptic version of K-S model

$$
\left\{\begin{array}{l}
\frac{\partial U}{\partial t}+\nabla(U \nabla S)=0 \\
-\Delta S=U
\end{array}\right.
$$

where coefficients $\mu$ and $\chi$ are removed by a scaling.

The validity of this version is supported by other interesting physical interpretations. It arises in statistical mechanics and in astrophysics as a model of the evolution of a cloud of self-gravitating particles in the mean field approximation (see $[1,2]$ and the references therein).

Comparing the parabolic-parabolic and the parabolic-elliptic models, authors in [2] expected that the evolution described by (26) might be faster than that for (1), especially when the diffusion of $S$ is rather slow compared to that of $U$. Moreover, the nonlinear effects for (26) should manifest themselves faster than for (26).

Otherwise, with the ad hoc decay conditions at infinity on $U$ and $S$, one should note that the chemical concentration gradient can be represented exactly by

$$
S(x, t)=-\int_{\mathbb{R}^{n}} E_{n}(x-y) U(y, t) d y
$$

where $E_{n}$ is the fundamental solution of the Laplacian in $\mathbb{R}^{n}$, a formula that can be used directly in the equation on $U$.

Let us focus on the investigation of blow up solutions and their behavior in a dimensional analysis. In the rest of this section, we will prove that under a certain scaling, (26) follows the inviscid Burgers' equation which results in development of singularity in finite time as Dirac $\delta$-function (amoebae aggregation) $[7,8]$. We summarize our results in the following theorem

Theorem 6.1. With a suitable initial data, nonnegative (i.e $U(x, 0)=U_{0}(x) \geq$ $0)$, chemotactic aggregation might occur in finite time at the proximity of the origin or on a ball in any space dimension. 
Remark 6.2. The arguments advanced here are actually independent on the dimension of the space in which aggregation occurs. This result would seem to contradict the diffusive nature of the model, but the fact that chemotaxis has some features of "negative diffusion" suggests the possibility of singular behavior.

\subsection{The one dimensional problem}

To begin with, we prove the following proposition

Proposition 6.3. The statements of Theorem 6.1 hold true in one dimension for some initial data.

Proof. In one dimension, we can rewrite (26) under the form

$$
\begin{array}{r}
\frac{\partial U}{\partial t}+\frac{\partial}{\partial x}\left[U \frac{\partial S}{\partial x}\right]=0 \\
\frac{\partial^{2} S}{\partial x^{2}}=-U
\end{array}
$$

Combining (27) and (28) we obtain

$$
\frac{\partial U}{\partial t}=-\frac{\partial}{\partial x}\left[U \frac{\partial S}{\partial x}\right]=\frac{\partial}{\partial x}\left[\frac{\partial^{2} S}{\partial x^{2}} \frac{\partial S}{\partial x}\right]
$$

From (28) we obtain

$$
\frac{\partial U}{\partial t}=-\frac{\partial^{3} S}{\partial t \partial x^{2}}
$$

(29) and (30) yields

$$
-\frac{\partial^{3} S}{\partial t \partial x^{2}}=\frac{\partial}{\partial x}\left[\frac{\partial^{2} S}{\partial x^{2}} \frac{\partial S}{\partial x}\right]
$$

With the transformation $V=\frac{\partial S}{\partial x},(31)$ takes the form

$$
\frac{\partial^{2} V}{\partial t \partial x}+\frac{\partial}{\partial x}\left[\frac{\partial V}{\partial x} V\right]=0
$$

Integrating (32), we obtain

$$
\frac{\partial V}{\partial t}+\frac{\partial V}{\partial x} V=0
$$


Finally

$$
\frac{\partial V}{\partial t}+\frac{\partial}{\partial x}\left[\frac{V^{2}}{2}\right]=0
$$

We amount to Burgers equation. So as soon as

$$
\frac{\partial V}{\partial x}(x, 0)=\frac{\partial^{2} S}{\partial x^{2}}(x, 0)=-U(x, 0)<0
$$

is satisfied at least for some values of $x$; then the classical theory of the conservation law predicts the apparition of the shock in finite time. So, there exist $\left(x^{*}, t^{*}\right)$ such that for $t>t^{*}, U=-\frac{\partial V}{\partial x}$ is a nonnegative measure, whose singular part is

$$
U_{1}(\cdot, t)=-\frac{\partial V}{\partial x} \mid(\cdot, t)=a(t) \delta(\cdot,-x(t))
$$

where $x=x(t)$ is Lipschitz curve and $a(t)$ is nonnegative bounded function. In particular, if we choose the initial data $S(x, 0)$ an odd-function and concave at the proximity of the origin. Then, $V=\frac{\partial S}{\partial x}$ is even decreasing at the proximity of the origin. So, for $t>t^{*}, U(\cdot, t)=a(t) \delta(\cdot)$.

Conclusion 6.4. For $n=1$, it's possible to have a collapse independently of the total mass as soon as the initial data $U(x, 0)$ does not become zero.

Remark 6.5. A similar idea has been used for another chemotactic model by J. Greenberg and W. alt [8].

With the same model of bacteria, i.e, to change (28) by $\frac{\partial^{2} S}{\partial x^{2}}=U$, we obtain

$$
\frac{\partial V}{\partial t}-\frac{\partial}{\partial x}\left[\frac{V^{2}}{2}\right]=0
$$

So, the formation of shocks will be if and only if there exist $x$ such that

$$
\frac{\partial V}{\partial x}=-U>0
$$

Basically, this is impossible because $u$ is nonnegative $(U \geq 0)$. Finally, within this context the chemotactic collapse never occurs in one dimension of space for the bacteria. 


\subsection{The multi-dimensional case $(n \geq 2)$}

In this case, we prove the following result

Proposition 6.6. The concentration in population of amoebae $U$ blows up as $\delta$ function on a ball.

Proof. Assuming that (26) holds radially symmetric solutions in n-space dimensions $(n \geq 2)$. Then $(26)$, in spherical coordinate, takes the form

$$
\begin{aligned}
\frac{\partial U}{\partial t}+\frac{1}{r^{n-1}} \frac{\partial}{\partial r}\left[r^{n-1} U \frac{\partial S}{\partial r}\right] & =0 \\
-\frac{1}{r^{n-1}} \frac{\partial}{\partial r}\left[r^{n-1} \frac{\partial S}{\partial r}\right] & =U
\end{aligned}
$$

Let us define $V:=r^{n-1} \frac{\partial S}{\partial r}$ then, on the one hand, from (34) we get

$$
\begin{array}{r}
-\frac{\partial}{\partial t}\left[r^{n-1} U\right]=\frac{\partial^{2} V}{\partial t \partial r} \\
U=-\frac{1}{r^{n-1}} \frac{\partial V}{\partial r}
\end{array}
$$

On the other hand, (33) yields

$$
-\frac{\partial}{\partial t}\left[r^{n-1} U\right]=\frac{\partial}{\partial r}(U V)
$$

From (35), (36) and (37) we deduce that

$$
\frac{\partial^{2} V}{\partial t \partial r}+\frac{\partial}{\partial r}\left[\frac{1}{r^{n-1}} V \frac{\partial V}{\partial r}\right]=0
$$

which yields, after integration

$$
\frac{\partial V}{\partial t}+\frac{1}{r^{n-1}} V \frac{\partial V}{\partial r}=0
$$

With the scaled variable

$$
\rho=\frac{r^{n}}{n} \Leftrightarrow \frac{\partial}{\partial r}=r^{n-1} \frac{\partial}{\partial \rho}
$$

(38) becomes

$$
\frac{\partial V}{\partial t}+V \frac{\partial V}{\partial \rho}=0
$$

which implies

$$
\frac{\partial V}{\partial t}+\frac{\partial}{\partial \rho}\left(\frac{V^{2}}{2}\right)=0
$$

We amount, once more, to Burgers' equation. 
Conclusion 6.7. If there exists $\rho>0$ such that $\frac{\partial V}{\partial \rho}(\rho, 0)<0$ then there exists a shock for $V$ since,

$$
\frac{\partial V}{\partial \rho}=\frac{1}{r^{n-1}} \frac{\partial}{\partial r}\left(r^{n-1} \frac{\partial S}{\partial r}\right)=-U
$$

and $U$ is nonnegative and not to be zero then $\frac{\partial V}{\partial \rho}<0$

Like for $n=1$, when there exists a shock for $V$ then, $U$ behaves as a deltafunction.

In particular, on $t=t^{*}$, the singular part of $U$ blows up as a delta-function (amoebae aggregation) on the ball with a radius $\rho=\rho^{*}$ in any space dimension.

\section{References}

[1] P. Biler and T. Nadzieja. A class of nonlocal parabolic problems occurring in statistical mechanics. Colloq. Math., 66:131-145, 1993.

[2] P. Biler and T. Nadzieja. Global and exploding solutions in a model of self-gravitating systems. Preprint 2002.

[3] J.T. Bonner. The cellular slime molds. Princeton University Press, Princeton, New Jersey, second edition., 1967.

[4] M.A.J. Chaplain. Avascular growth, angiogenesis and vascular growth in solid tumors : the mathematical modelling of the stages of tumor development. Math. Comput. Modelling, 23:47-87, 1996.

[5] S. Childress and J.K. Percus. Nonlinear aspects of chemotaxis. Math. Biosci., 66:217-237, 1981.

[6] L. Corrias M.P. Biler and J. Dolbeault. Large mass self-similar solutions of the parabolic-parabolic kellersegel model of chemotaxis. J. Math. Biol., 2010 .

[7] A.F. Filipov. Differential equation with discontinous right-hand side. Trans. Amer. Math. Soc., 42:199-231, 1964.

[8] J.M. Greenberg and W. Alt. Stability results for a diffusion equation with functional drift approximating a chemotaxis model. Trans. Amer. Math. Soc., 300:235-258, 1987.

[9] E. Hopf. The partial differential equation $u_{t}+u u_{x x}=\mu u u_{x x}$. Com. Pur. Appl. Math., 13:201-230, 1950. 
[10] D. Horstmann. From 1970 until present: the keller-segel model in chemotaxis and its consequences. Max-Planck-Institut Preprint n.3., 2003.

[11] E.F. Keller and L.A. Segel. Initiation of slime mold aggregation viewed as an instability. J. Theor. Biol., 26:399-415, 1970.

[12] E.F. Keller and L.A. Segel. Model for chemotaxis. J. Theor. Biol., 30:225234, 1971.

[13] E.F. Keller and L.A. Segel. Travelling bands of chemotactic bacteria, a theoretical analysis. J. Theor. Biol., 30:235-248, 1971.

[14] Y. Naito. Asymptotically self-similar solutions for the parabolic system modelling chemotaxis. Banach Center Publ. Polish Acad. Sci., Warsaw, 74:149-160, 2006.

[15] K.J. Painter T. Hillen. A user's guide to PDE models for chemotaxis. J. Math. Biol., 58:183-217, 2009.

[16] C.S. Patlak. Random walk with persistence and external bias. Bull. of Math. Biophys., 15:311-338, 1953.

[17] B. Perthame L. Corrias and H. Zaag. A chemotaxis model motivated by angiogenesis. C. R. Acad. Sci. Paris, Ser. I, 336:141-146, 2003.

[18] M. Rascle and C. Ziti. Finite time blow-up in some models of chemotaxis. J. Math. Biol., 33:388-414, 1995.

[19] C. Ziti. Résolution numrique d'un système non strictement hyperbolique modélisant le comportement d'une population bactérienne. PhD thesis, C.R.A.S., 1994.

Saïd HILMI,

Department of Mathematics,

University of Moulay Ismail,

Faculty of Sciences of Meknès,

BP 11201 Zitoune, Meknès 50000, Morocco.

Email: hilmi_said@hotmail.com

Chérif ZITI,

Department of Mathematics,

University of Moulay Ismail,

Faculty of Sciences of Meknès,

BP 11201 Zitoune, Meknès 50000, Morocco.

Email: chziti@gmail.com 